\title{
Primary Malignant Melanoma of the Vagina: Report of Two Cases and Review of the Literature
}

\author{
Guler Yavas $^{1^{*}}$, Irem Oner ${ }^{2}$, Cagdas Yavas ${ }^{1}$, Erdem Sen $^{2}$, Pinar Karabagli $^{3}$, Cetin Celik ${ }^{4}$ and Ozlem Ata ${ }^{2}$ \\ ${ }^{1}$ Selcuk University, Department of Radiation Oncology, Konya, Turkey \\ ${ }^{2}$ Selcuk University, Department of Medical Oncology, Konya, Turkey \\ ${ }^{3}$ Selcuk University, Department of Pathology, Konya, Turkey \\ ${ }^{4}$ Selcuk University, Department of Obstetrics and Gynecology, Division of Gynecologic Oncology, Konya, Turkey
}

*Corresponding Author: Guler Yavas, Selcuk University Faculty of Medicine, Department of Radiation Oncology Konya, Turkey, Selcuklu, Konya, Turkey, Tel: +90-332-224-40-85; Fax: +90-332-2417791; E-mail: guler.aydinyavas@gmail.com

Received: 20 July 2017; Accepted: 27 July 2017; Published: 01 August 2017

\begin{abstract}
Vaginal malignant melanoma is a rare form of mucosal melanoma, and accounts for only $<1 \%$ of all melanomas and $1.6 \%$ of female genital tract melanomas. Due to the rarity of the disease, currently, no standard treatment protocol has been established for the treatment of vaginal melanomas. Unfortunately, vaginal melanomas are often only diagnosed at an advanced stage, and treatment options include local excision with wide margins, radical surgery, radiotherapy, chemotherapy, and immunotherapy. Despite these aggressive treatment approach, the prognosis of vaginal melanoma is poor, and the 5-year overall survival rate is $0-25 \%$. Since patients with vaginal melanoma ultimately develop distant metastatic disease regardless of the primary treatment approach; patient preference and quality-of-life considerations are critical factors in determining initial management. Herein we report two cases of vaginal melanoma. The first case was staged as T4bN0M0 according to the American Joint Committee on Cancer (AJCC) classification system. She denied surgery therefore underwent definitive radiotherapy. The patient is on routine follow-up, and now after 13 months of the completion of the treatment, she is still free of disease. The second case was T4bN1M0 according to AJCC classification system and underwent wide local excision with vaginectomy and bilateral inguinal lymph node dissection followed by adjuvant radiotherapy and immunotherapy. She is still free of disease for 34 months. In these report we discussed the optimal treatment strategy for vaginal melanomas by the light of available literature.
\end{abstract}


Keywords: Brachytherapy; Immunotherapy; Radiotherapy; Surgery; Vaginal melanoma

\section{Introduction}

Mucosal melanomas are rare and account for approximately $1 \%$ of all melanomas [1]. Mucosal melanomas generally carry a worse prognosis than those arising from cutaneous sites. Since the unique biology and clinical challenges of mucosal melanoma arising from each anatomic location, our understanding of these malignancies and their optimal management remains limited [2].

Vaginal malignant melanoma is a rare form of mucosal melanoma, and accounts for only $<1 \%$ of all melanomas, $1.6 \%$ of female genital tract melanomas; and $\sim 5 \%$ of all vaginal malignant tumors [1,3]. The age of onset of vaginal melanoma has been reported to range from 38 to 90 years, with most patients being diagnosed between the ages of 60 and 80 years. Patients commonly complain of vaginal bleeding, vaginal discharge or a palpable mass [1]. No staging system has been demonstrated to be a useful predictor of prognosis in vaginal melanoma, although tumor size $(<3 \mathrm{~cm}$ versus $\geq 3 \mathrm{~cm})$, has been shown to predict survival in some series $[4,5]$. In the absence of such a prognostic staging system for this disease, it would seem reasonable to use the American Joint Committee on Cancer (AJCC) staging system in vaginal melanoma for the purposes of standardization [2]. In this system, stages I, II, and III correspond to clinically localized disease, regional lymph node involvement, and distant metastases, respectively. Currently, no standard treatment protocol has been established for the treatment of vaginal melanomas. Unfortunately, vaginal melanomas are often only diagnosed at an advanced stage, and treatment options include local excision with wide margins, radical surgery, radiotherapy, chemotherapy, and immunotherapy. Since patients with vaginal melanoma ultimately develop distant metastatic disease regardless of the primary treatment approach; patient preference and quality-of-life considerations are critical factors in determining initial management [2]. Despite aggressive treatment approach in a multidisciplinary team, the prognosis of vaginal melanoma is poor, and the 5 -year overall survival rate is $0-25 \%[2,6,7]$. Herein we present two cases of vaginal melanoma.

\section{Case Report-1}

A 66-year-old woman presented with a 4-month history of feeling a mass in the vagina. On physical examination, a nodular pigmented mass noted beginning from the anterior vaginal fornix and extending through the whole anterior vaginal wall. Histopathological examination of the mass revealed a nodular melanoma (Figure 1). The diameter of the tumor was $3 \times 2.2 \mathrm{~cm}$; and the thickness of the tumor was $11 \mathrm{~mm}$. There was ulceration in the tumor. The mitotic rate was $>1 / \mathrm{mm}^{2}$. There was no lymphovascular invasion and perineural invasion. The tumor was immonureactive for both HMB 45 and S-100 (Figure 2). There wasn't any palpable lymph node in bilateral inguinal area. The patient had no distant metastasis with routine work-up. According to AJCC classification system the patient was staged as T4bNOM0, stage IIC. Although we offered initial surgery, the patient refused surgery. Therefore she underwent definitive radiotherapy. We performed external radiotherapy to pelvis+bilateral groin chains and vagina using 3dimensional conformal radiotherapy to a dose of $50 \mathrm{~Gy}$ in 25 daily fractions of 2 Gy each. After external radiotherapy, the patient underwent vaginal brachytherapy to a dose of $28 \mathrm{~Gy}$ in 4 fractions over 2 weeks. Treatment was given without interruption. She had grade 2 cystitis and grade 2 diarrhea which respond to medical treatments. 
In addition she had grade $2-3$ radiation dermatitis in vulvo-vaginal area. She patient is on routine follow-up, and now after 13 months of follow-up she is still free of disease.

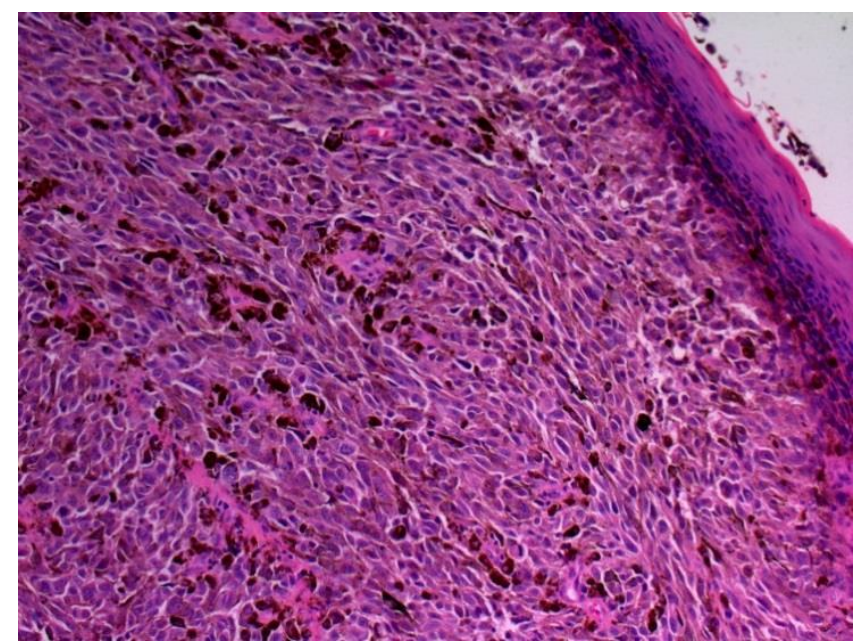

Figure 1: Histopathological examination of the mass revealed a nodular melanoma (H\&E X200).

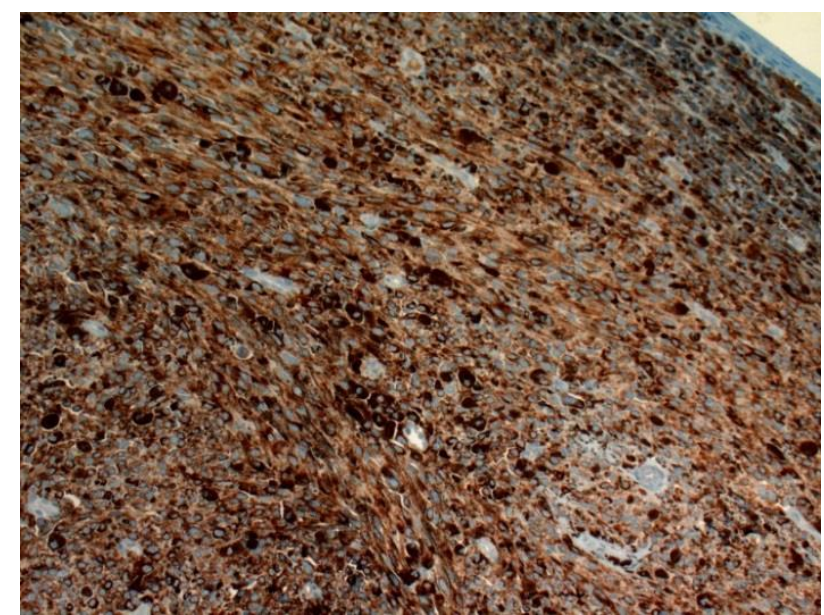

(a)

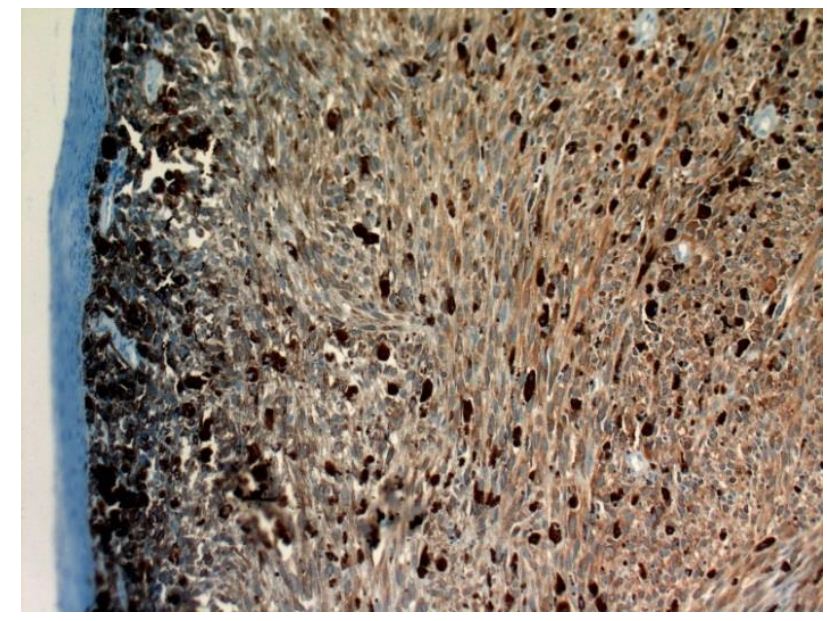

(b)

Figure 2: Immunohistochemical HMB 45 staining of the tumor (a); and immunohistochemical S-100 staining of the tumor (x200) (b). Neoplasm composed of spindle nucleus, prominent macronucleoli cells containing diffuse melanin pigment in nodular pattern. Positive immunoreactivity was seen with S-100 and HMB 45. 


\section{Case Report-2}

A 67-year-old woman presented with history of abnormal genital bleeding for last 3 months. On gynecological examination, a brown colored nodular mass with a subtle irregular surface at lower $1 / 3$ postero-lateral vaginal wall was noted. Bilateral parametria were free and rectum was normal with bimanual examination and there was a palpable inguinal lymphadenopathy on the right inguinal area. An excisional biopsy from the mass was done and histopathological examination revealed malign melanoma. Therefore metastatic work-up was done. Fluorine-18fluorodeoxy-glucose positron emission tomography-computed tomography (PET-CT) revealed that there was no distant metastasis. Wide local excision with vaginectomy and bilateral inguinal lymph node dissection was done. During operation there was a urethral infiltration of the tumor; therefore inferior $1 / 3$ part of the urethra was also resected. Histopathological examination was compatible with malign melanoma, which was $5 \times 5 \mathrm{~cm}$ in diameter. The tumor was immonureactive for both HMB 45 and S-100. There was extensive ulceration. The thickness of the tumor was $13 \mathrm{~mm}$. There was a 1 out of 11 metastatic lymph node on the right inguinal side; however there wasn't any extra capsular invasion. The metastatic lymph node was $0.9 \mathrm{~cm}$ in diameter (Figure 3). Although there were lentiginous changes in the pathological material there was no residual tumor. According to AJCC classification system, the patient was staged as stage III (T4bN1bM0). The patient underwent adjuvant radiotherapy (50.4 Gy in 28 fractions; $1.8 \mathrm{~Gy}$ per fraction) to the primary tumor and bilateral inguinal lymph nodes in frog-leg position followed by a $30 \mathrm{MU}$ interferon- $\alpha$ treatment every other day for 2 years. Now we completed the planned adjuvant treatment and still patient is free of disease for 34 months.

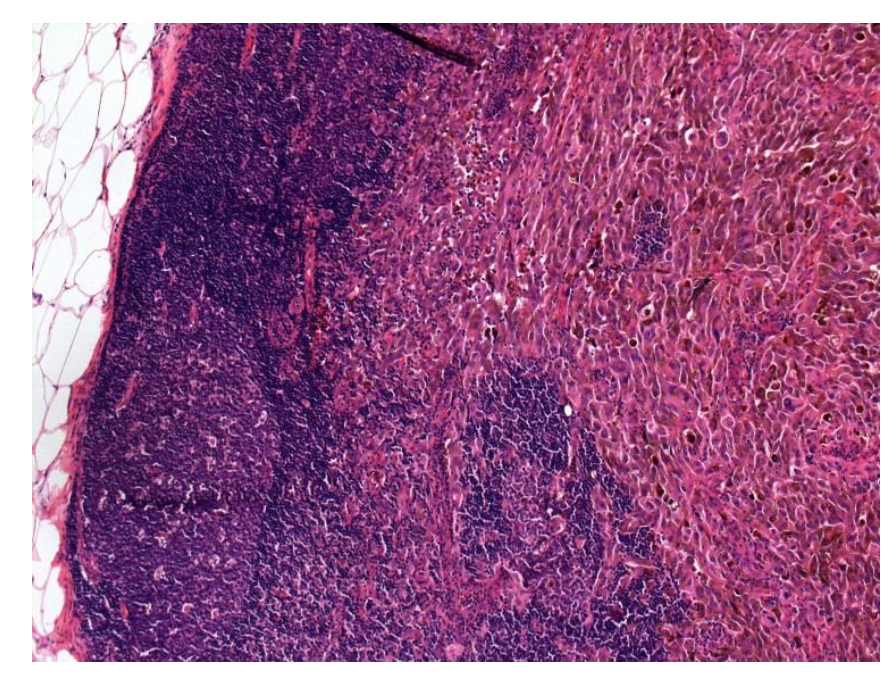

Figure 3: Melanoma metastasis is seen in the lymph node (x100 H\&E).

\section{Discussion}

Malignant melanoma arising from the vaginal mucosa is thought to originate from mucosal melanocytes in regions of melanosis or from atypical melanocytic hyperplasia [8]. In 3\% of healthy women, melanocytes can be found in the basal portion of the vaginal epidermis, as an embryological remnant of neural crest cells [6,9]. Primary malignant vaginal melanoma is one such type of mucosal malignant melanoma that is very rare and very aggressive in nature $[1-3,9]$. The first case of primary malignant vaginal melanoma was reported in 1887 and modern literature 
has noted about 500 cases, globally [8]. To date there is no clear consensus regarding to treatment of vaginal melanomas. We reported two-cases of vaginal melanoma who underwent either definitive or adjuvant radiotherapy. Huang and colleagues retrospectively analyzed the clinical characteristics of and prognostic factors of 31 patients with the diagnosis of primary vaginal melanoma [1]. The median age was 58 years (range, 18 to 73 years), and the main symptoms reported were vaginal bleeding and vaginal discharge. In our cases, one of the patients was 66 years old, and admitted with a feeling of vaginal mass; and the other was 67 years old and presented with vaginal bleeding. In Huang and colleagues report, surgery was performed on 22 patients. Two of the patients did not receive any treatment. Neoadjuvant chemotherapy was administered to 6 patients, and 1 patient underwent brachytherapy prior to surgery. Following surgery, 16 patients received chemotherapy and 2 patients underwent chemoradiation therapy. Chemotherapy alone without surgery was used to treat 7 patients, 4 of whom received immunotherapy together with chemotherapy. The disease recurred in $35.5 \%$ cases, 3 of which $(27.3 \%)$ involved local recurrence, 7 involved (63.6\%) distant recurrence, and 1 involved (9.1\%) both local and distant recurrence. The 5-year overall survival rate was 32.3\%. Macroscopic tumor growth and treatment method were found as prognostic factors for primary malignant melanoma of the vagina. It is clear from this retrospective analysis by Huang and colleagues that the treatment options not standard and differs both due to the nature of the disease and choose of the patients. Similarly, in our cases one the patients refused surgery; therefore we planned definitive radiotherapy, and still free of disease 13 months after the completion of treatment. The other case underwent radical surgery followed by radiotherapy and immunotherapy. In second patient there are still no signs or symptoms of disease for 34 months.

Tasaka et al. reported a total 5 patients with vaginal melanoma, treated at the Department of Gynecology of Osaka City University Hospital [3]. All the cases presented with abnormal genital bleeding as the main complaint. Notably, in 3 of the 5 cases, the tumors appeared as non-pigmented polyps. In all 5 cases, local resection was performed as primary treatment and 4 out of 5 patients received adjuvant immunotherapy. After a year all of the patients recurred, and the overall survival and disease free survivals were reported as 419 days; and 177 days respectively. Kalampokas and colleagues reported a case of vaginal melanoma who underwent wide local excision with at least $1.5 \mathrm{~cm}$ free margins [9]. Since the patient refused any additional treatment such as radiotherapy or chemotherapy she did not receive adjuvant therapy. Twelve months after the initial diagnosis and follow-up there remain no signs of disease recurrence.

Many authors think that even in the most minimally invasive and small lesions of vagina, radical surgical resection must be performed. Otherwise, the local recurrence is claimed to be as high as 80\% [6, 10]. Geisler et al. advised primary pelvic exenteration for vaginal melanoma over $3 \mathrm{~mm}$ of invasion and showed that 50\% 5-year survival rate might be obtained if the pelvic nodes were free of metastases [11]. Chung and Ariel, with separate series of 19 and 48 patients, respectively, defend the opinion that the appropriate management of this disease requires radical extirpation of the vagina supplemented by dissection of the regional lymphatics [10]. Therefore it seems that standard management of vaginal melanoma should involve surgery if possible. Achieving negative margins in these cases can be difficult without pelvic exenteration given the high frequency of multifocality and anatomic constraints. However, the impact of such radical surgery upon survival when compared with more conservative procedures including definitive or adjuvant radiotherapy with limited surgery is still unclear. Thus, surgery may be combined 
with RT in select cases [2]. Therefore in our cases we preferred initial surgery for both; however since one of the refused surgery we performed definitive radiotherapy.

Radiotherapy may have role either as an adjuvant following surgery or when surgery is not feasible due to the disease or patient related factors. In spite of aggressive surgery it was known that in 29-79\% cases of vaginal melanoma experience local recurrence [2, 12, 13]. Radiotherapy has been used in the adjuvant setting, with limited data demonstrating improvement in regional control with no benefit in overall survival [2, 8, 12 ]. Nevertheless some centers recommend routine postoperative RT for those with palpable lymph nodes or the finding of extracapsular extension following wide local excision and lymph node dissection. Primary radiotherapy may be an option for patients who refused surgery, and who are not candidates for resection or when an adequate resection margin is not possible [12]. Optimal dosing and fractionation schedules have not been established for RT either as primary therapy or as an adjuvant to surgery. Given the high rates of distant metastases, chemotherapy has been used, either alone or in conjunction with radiotherapy; however there are no consistent recommendations with regard to the benefits of chemotherapy and immunotherapy [8].

In conclusion due to the rarity of vaginal melanoma there is no consensus regarding to optimal treatment modality. Available data demonstrates that if possible the initial surgery is the best option; however still there is not enough evidence supporting an aggressive surgery. Therefore more conservative surgery with adjuvant therapies including immunotherapy, chemotherapy and radiotherapy may be reasonable. Further studies with more patients and longer follow-up period are warranted in order to define the best treatment strategy.

\section{Conflict of Interest}

The authors declare that they have no conflict of interest

\section{References}

1. Huang Q, Huang H, Wan T, et al. Clinical outcome of 31 patients with primary malignant melanoma of the vagina. J Gynecol Oncol 24 (2013): 330-335.

2. Carvajal RD, Hamid O, Charlotte Ariyan C. Mucosal melanoma (2017).

3. Tasaka R, Fukuda T, Wada T, et al. A retrospective clinical analysis of 5 cases of vaginal melanoma. Mol Clin Oncol 6 (2017): 373-376.

4. Buchanan DJ, Schlaerth J, Kurosaki T. Primary vaginal melanoma: thirteen-year disease-free survival after wide local excision and review of recent literature. Am J Obstet Gynecol 178 (1998): 1177-1184.

5. Petru E, Nagele F, Czerwenka K, et al. Primary malignant melanoma of the vagina: long-term remission following radiation therapy. Gynecol Oncol 70 (1998): 23-26.

6. Gökaslan H, Sişmanoğlu A, Pekin T, et al. Primary malignant melanoma of the vagina: a case report and review of the current treatment options. Eur J Obstet Gynecol Reprod Biol 121 (2005): 243-248.

7. Weinstock MA. Malignant melanoma of the vulva and vagina in the United States: patterns of incidence and population-based estimates of survival. Am J Obstet Gynecol 171 (1994): 1225. 
8. Kang J, Viswanathan AN. Vaginal Cancer (chapter 72). In Principles and Practice Radiation Oncology ed: Halperin EC, Wazer DE, Perez CA, et al. $6^{\text {th }}$ Edn. Walters Kluwer/Lippincott Williams \&Wilkins. Philedelphia, USA (2013): 1479.

9. Kalampokas E, Kalampokas T, Damaskos C. Primary Vaginal Melanoma, A Rare and Aggressive Entity. A Case Report and Review of the Literature. In Vivo. 31 (2017): 133-139.

10. Irvine WP, Bliss SA, Rice LW, et al. Malignant melanoma of the vagina and locoregional control: radical surgery revisited. Gynecol Oncol 71 (1998): 476-480.

11. Geisler JP, Look KY, Moore DA, et al. Pelvic exenteration for malignant melanoma of the vagina or urethra with over $3 \mathrm{~mm}$ of invasion. Gynecol Oncol 59 (1995): 338-341.

12. Krengli M, Masini L, Kaanders JH, et al. Radiotherapy in the treatment of mucosal melanoma of the upper aerodigestive tract: analysis of 74 cases. A Rare Cancer Network study. Int J Radiat Oncol Biol Phys 65 (2006): 751.

13. Temam S, Mamelle G, Marandas P, et al. Postoperative radiotherapy for primary mucosal melanoma of the head and neck. Cancer 103 (2005): 313.

This article is an open access article distributed under the terms and conditions of the

Creative Commons Attribution (CC-BY) license 4.0 